\title{
Mechanical Characterization of CoCrMo Alloys Consisting of Different Palladium Ratios Produced by Investment Casting Method
}

\author{
O. SAhin*, S. Uzunoglu, E. SAhin \\ Mustafa Kemal University, Science and Art Faculty, Micro/Nanomechanic Characterization Laboratory, \\ Hatay 31034, Turkey

\begin{abstract}
CoCrMo alloys are one of the most commonly used materials for hip arthroplasty, knee and dental because of its mechanical properties, corrosion resistance, wear resistance and biocompatibility. In this study, CoCrMo alloys consisting of 1.68 to $4.33 \% \mathrm{Pd}$ are produced by investment casting process under argon atmosphere. The microstructures and mechanical properties of CoCrMo alloy were studied using X-ray diffraction, optical microscopy, scanning electron microscopy, Knoop indentation hardness tests, focusing on the influences on the different palladium additives. The measured microhardness values of CoCrMo alloys having different palladium ratio are seen to be load-dependent. The observed load dependence was rationalized using the Hays-Kendall model and it was found that the resultant load-independent hardness decreases with increase of palladium ratios. As a results, microhardness decreases with increase of palladium amount.
\end{abstract}

DOI: 10.12693/APhysPolA.128.B-149

PACS: $62.20 .-\mathrm{x}, 61.66 . \mathrm{Dk}$

\section{Introduction}

Investment castings made out of $\mathrm{Co}-\mathrm{Cr}-\mathrm{Mo}$ alloys have been widely employed in the manufacture of surgical prostheses due to their high corrosion resistance and adequate mechanical properties [1]. However, in the as-cast condition, preferential distributions of extensive interdendritic and grain boundary carbides significantly affect the alloy ductility. Thermal processing has been commonly used in Co alloys to modify the potential carbide precipitation reactions. Various carbide microstructures [2] can be produced which in turn might lead to improved alloy strength. In particular, the development of stacking faults and twins during alloy heat treating seems to play a key role in the resultant carbide distribution and morphology [3].

Over the years CoCrMo has demonstrated a remarkable level of versatility and durability as an orthopaedic implant material. This alloys are widely employed in the manufacture of prostheses for hip and knee joints due to their biocompatibility and their superior mechanical properties such as: high elastic modulus, high tensile strength, high wear resistance and high corrosion resistance.

In the literature $\mathrm{Co}-\mathrm{Cr}$ base alloys were in the cast of dental implants. In order to achieve better performances and to improve their relatively poor ductility the addition of different alloying elements has been intensively studied [4]. In the $\mathrm{Co}-\mathrm{Cr}-\mathrm{Mo}$ ternary phase diagram, the crystal structure of the $\mathrm{Co}-\mathrm{Cr}-\mathrm{Mo}$ alloys will transform from the phase $\gamma$ which is stable at high temperatures to

*corresponding author; e-mail: sduosman@gmail.com the $\varepsilon$ phase (hcp) which is stable at room temperature martensitically or diffusionally depending on the cooling rate. One can absolutely say that additives influence the mechanical properties of the materials. In addition, the addition of a fourth element to $\mathrm{Co}-\mathrm{Cr}-\mathrm{Mo}$ alloys is one of the effective methods, owing to stabilize the $\gamma$ phase. It has been reported that $\mathrm{Ni}, \mathrm{C}$, and $\mathrm{N}$ addition stabilize the phase in $\mathrm{Co}-\mathrm{Cr}-\mathrm{Mo}$ alloys [5]. The purpose of this article is to present the results of an experimental study of the effect of the palladium addition on the mechanical properties of the CoCrMo alloys.

\section{Experimental procedure}

Melting temperature of CoCrMo alloy was selected in the range of $1440-1520^{\circ} \mathrm{C}$. The composition by mass\% was $30 \mathrm{Cr}, 5.7 \mathrm{Mo}, 0.8 \mathrm{Ni}, 0.02 \mathrm{C}$, with Co making up the balance. The cast alloy was melted in a induction casting furnace under an argon atmosphere. In the furnace, the metal inside the crucible is heated by induction. Silica-based crucibles were used for the melt alloy and were pre-heated to $420^{\circ} \mathrm{C}$ to eliminate humidity. In centrifugal casting, the molten metal fills the hollowed investment mold by centrifugal force. The mold is fixed horizontally at the end of the arm. When the metallic bath is at the casting temperature the casting arm begins to rotate and the metal is poured inside the mold. The casting pressure is about 21 to 26 bar. After casting, the investment cooled in air removed and metallic micro components were cleaned. To examine the surfaces, investigations were performed by a FEI Quanta FEG 250 scanning electron microscope (SEM). X-ray diffraction (XRD) (Rigaku Co, Japan) was carried out to analyze the phase compositions in a continuous-scanning mode with $\mathrm{Cu} K_{\alpha}$ radiation.

Microhardness measurements of the alloys were performed at room temperature using the Knoop indenter. 
Damage on the surface due to mechanization is removed mechanically by grinding 1200 and 2400 grit, and then polishing on 6,3 , and $1 \mu \mathrm{m}$ diamond lap wheels. Indentation tests loads ranging from 0.245 to $9.8 \mathrm{~N}$ and loading time of $15 \mathrm{~s}$ were used to measure the diagonals of the indentations. In view of the scatter of the microhardness data, the hardness value was a mean of at least six measurements under the same condition.

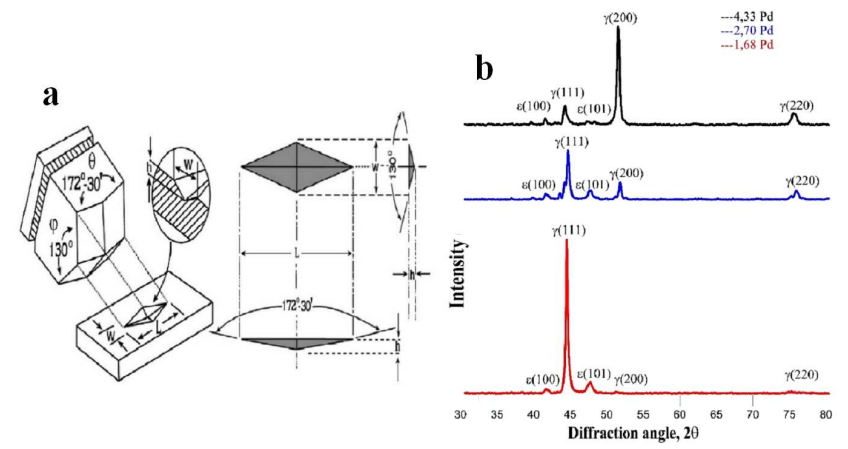

Fig. 1. (a) Knoop indentation and indentation diagonal length, (b) XRD data for CoCrMoPd alloys.

The Knoop hardness test used a lozenge-based pyramid with the angle $\theta$ between the two opposite faces being $172.5^{\circ}$ and the angle $\varphi$ between the other two being $130^{\circ}$ (Fig. 1a). Calculation of the Knoop hardness number considers the projected area of contact in the plane of the material. The projected area is calculated using the length of the indent by knowing the theoretical relationship between the length and the width of the impression. The Knoop hardness number $\left(H_{\mathrm{N}}\right)$ is calculated as follows:

$H_{\mathrm{N}}=\frac{P_{\max }}{A}=\frac{P}{L^{2} \tan (\varphi / 2) / 2 \tan (\theta / 2)}\left(=14.229 \frac{P}{L^{2}}\right)$,

where $H_{\mathrm{N}}$ is expressed in $\mathrm{MPa}$, if $P$ the applied load is in $\mathrm{N}$ and $L$ is the large diagonal of the indent in $\mathrm{mm}$. $A$ represents the projected area of contact, and 14.229 is a constant of geometrical factor for the diamond pyramid [6]. The indentation test procedure is similar to the ASTM standard E384 (Standard Test method for Microhardness of Materials, ASTM Designation E384, 1991. Book of ASTM Standard, Part 3. American Society for Testing and Materials, Philadelphia, PA).

\section{Results and discussions}

Prior to XRD measurement, the surfaces of the three different samples were ground with 2400 grit $\mathrm{SiC}$ paper, and then sequentially polished with $1 \mu \mathrm{m} \mathrm{Al}{ }_{2} \mathrm{O}_{3}, 0.3 \mu \mathrm{m}$ $\mathrm{Al}_{2} \mathrm{O}_{3}$, and a colloidal $\mathrm{SiO}_{2}$ suspension to form mirrorlike surfaces. The polished samples were ultrasonically rinsed in distilled water, alcohol, and acetone. The X-ray diffraction (XRD) patterns of the CoCrMo alloys having different $\mathrm{Pd}$ is shown in Fig. 1. For all the samples, the structure appears to be a mixture of $\gamma$-CoCr and $\varepsilon$ - $\mathrm{CoCr}$ cobalt parent structures with solid solution chromium and molybdenum; and there is a higher concentration of the $\gamma$-CoCr phase or ratio of $\gamma / \varepsilon$. As can be seen in this figure, $\gamma$ phase is predominant with a small amount of the $\varepsilon$ phase. When the $\mathrm{Pd}$ content in the CoCrMo alloy further increases, the intensities of the (111) and (101) peaks decrease evidently. Therefore, it can be concluded that the alloy formation mechanism and hence the crystallinity change with $\mathrm{Pd}$ concentration [7].

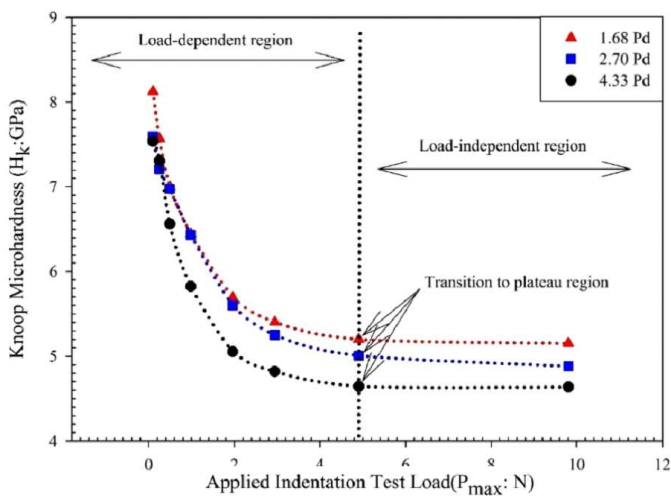

Fig. 2. Variation of the Knoop microhardness with the applied indentation test load.

Figure 2 shows the variation of Knoop microhardness as a function of applied test loads ranging from 0.245 to $9.8 \mathrm{~N}$ for CoCrMo alloy. The microhardness decreases with the increase of applied indentation test load, and then reaches a saturation value at higher loads. Thus the entire hardness profile consists of two regimes: loaddependent and load-independent regimes. The behaviour of microhardness in both the regimes could be explained to take into account the nature of the indented surface. In the low load regime the indenter penetrates into only the upper surface layer of the crystals. There is a sharp fall of the microhardness value in the low-load region depending on the strain distribution of the upper surface layer. The depth of the penetration of the indenter increases with the increase of the load. Both the effects of the inner layer and surface layer contribute to the hardness, varying nonlinearly with load. This type of behaviour is called indentation size effect (ISE) [7, 8].
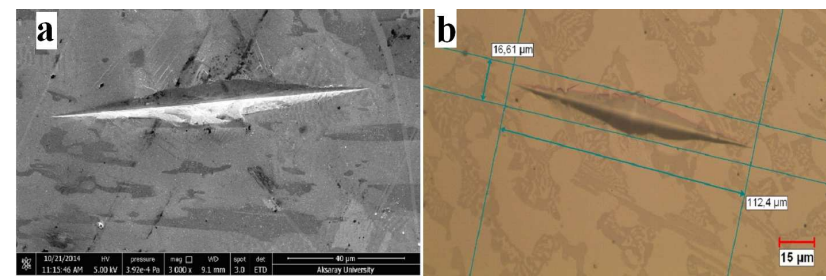

Fig. 3. (a) A typical SEM micrograph of CoCrMoPd alloy, (b) a typical optical micrograph of $\mathrm{CoCrMoPd}$ alloy.

In order to describe the ISE behaviour of different materials, several models for the relation between the applied indentation test load and the indentation diagonal length have been reported in the literature $[9,10]$. One of the model, the Hays-Kendall one, is presented in the next section, and it is applied to analyze the 
microhardness data of the CoCrMo alloy having different $\mathrm{Pd}$ ratio. In addition, the SEM and optical micrograph of CoCrMo alloy can be seen in Fig. 3a and b. From this figures the sample surfaces are homogeneous and without cracks. Furthermore, we believe that there is no significant surface finishing effect since the shape of the Knoop indenter is exactly the same as the indentation observed on the sample surface. Therefore, it is judged to be unnecessary to discuss the effect of microcracking on the microhardness measurements in our study.

\subsection{Load-independent microhardness calculation for CoCrMo alloys using Hays-Kendall model}

The Hays-Kendall model was suggested by Hays and Kendall (HK) [11]. They proposed that there exists a minimum applied test load $W$ (test specimen resistance) necessary to initiate plastic deformation and below which only elastic deformation occurs. They introduced an effective indentation load, $P_{\text {eff }}=P_{\max }-W$, and proposed the following relationship between the applied load and the resultant indentation size:

$$
P_{\max }-W=C_{1} L^{2},
$$

where $C_{1}$ is the load-independent hardness constant calculated by HK approach for a given sample. From the Eq. (2), a plot of $P_{\max }$ versus $L^{2}$ would yield a straight line. Such plot for CoCrMo alloys having different $\mathrm{Pd}$ ratio considered in the present study is shown in Fig. 4.

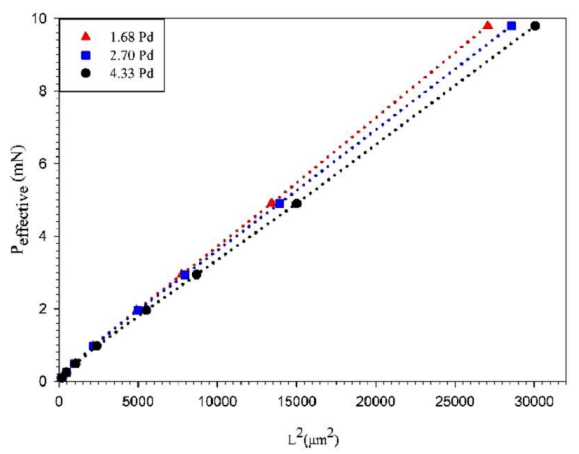

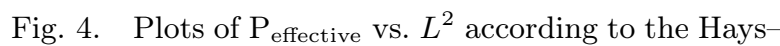
Kendall model.

The $C_{1}$ and $W$ values in Eq. (2), are given in Table. The correlation coefficient for examined materials, $r^{2}$ are 0.999 , implying that Eq. (2) provides a satisfactory description of the indentation data for the materials.

TABLE

Best-fit results of the parameters in Eqs. (2), (3).

\begin{tabular}{l|c|c|c|c|c}
\hline \hline Pd ratio & $\begin{array}{c}W \\
{[\mathrm{~N}]}\end{array}$ & $\begin{array}{c}C_{1} \\
{\left[\mathrm{~N} / \mu \mathrm{m}^{2}\right]}\end{array}$ & $\begin{array}{c}H_{\mathrm{HK}} \\
{[\mathrm{GPa}]}\end{array}$ & $\begin{array}{c}H_{\mathrm{LI}} \\
\text { Plateau values } \\
{[\mathrm{GPa}]}\end{array}$ & $r^{2}$ \\
\hline $1.68 \mathrm{Pd}$ & 0.1316 & $3.578 \times 10^{-4}$ & 5.090 & 5.153 & 0.999 \\
$2.70 \mathrm{Pd}$ & 0.1647 & $3.391 \times 10^{-4}$ & 4.825 & 4.882 & 0.999 \\
$4.33 \mathrm{Pd}$ & 0.1294 & $3.215 \times 10^{-4}$ & 4.575 & 4.637 & 0.999
\end{tabular}

According to the HK approximation, load independent microhardness, $H_{\mathrm{HK}}$, can be calculated as

$$
H_{\mathrm{HK}}=14.229 C_{1} .
$$

The load-independent microhardness of the examined CoCrMo aloys were calculated using Eq. (3) and the best fit values of $C_{1}$ are listed in Table.

In the present study, Fig. 2 shows the transition point (about $4.9 \mathrm{~N}$ ) and corresponding intrinsic hardness values $5.153,4.882$, and $4.637 \mathrm{GPa}$ for $1.68 \mathrm{Pd}, 2.70 \mathrm{Pd}$, and $4.33 \mathrm{Pd}$, respectively. From Table one can see that the load-independent microhardness values calculated in the HK model are very close to the plateau values. As a result, one can conclude that the Hays-Kendall approach is appropriate for describing the ISE behaviour observed in CoCrMo alloys.

\section{Conclusion}

From the above observations, the following conclusions can be drawn:

1. The measured microhardness values of $\mathrm{CoCrMoPd}$ alloys are seen to be load-dependent.

2. The variation of $H_{\mathrm{K}}$ follows the normal ISE trend, i.e., a decrease in $\mathrm{Hv}$ with an increase load in the lowload region beyond which it becomes relatively constant. This type of variation in $H_{\mathrm{K}}$ can be explained by HaysKendall model. Load-independent Knoop hardness values are $5.153,4.882$, and $4.637 \mathrm{GPa}$ for $1.68 \mathrm{Pd}, 2.70 \mathrm{Pd}$, and $4.33 \mathrm{Pd}$, respectively.

3. The microhardness decreases with increase of palladium amount.

\section{Acknowledgments}

The financial support from research foundation of Mustafa Kemal University (grant no. 08 F 0501 and 8481) is also gratefully acknowledged.

\section{References}

[1] P.C. Noble, Met. Forum 6, 59 (1983).

[2] R.N.J. Taylor, R.B. Waterhouse, J. Mater. Sci. 18, 3265 (1983).

[3] C. Montero-Ocampo, M. Talavera, H. Lopez, Metall. Mater. Trans. 30A, 611 (1999).

[4] S. Longquan, D.O. Northwood, C. Zhengwang, J. Mater. Sci. 28, 1312 (1993).

[5] S.-H. Lee, E. Takahashi, N. Nomura, A. Chiba, Mater. Trans. 46, 1790 (2005).

[6] D. Chicot, D. Mercier, F. Roudet, K. Silva, M.H. Staia, J. Lesage, J. Europ. Ceram. Soc. 27, 1905 (2007).

[7] O. Sahin, A. Riza Tuncdemir, H.A. Cetinkara, H. Salih Guder, E. Sahin, Chin. Phys. Lett. 28, 126201 (2011).

[8] O. Sahin, O. Uzun, U. Kolemen, N. Ucar, Mater. Character. 58, 197 (2007).

[9] K. Sangwal, B. Surowska, P. Blaziak, Mater. Chem. Phys. 80, 428 (2003).

[10] J. Gong, H. Miao, Z. Zhao, Z. Guan, Mater. Sci. Eng. A 303, 179 (2001).

[11] C. Hays, E.G. Kendall, Metallography 6, 275 (1973). 